\title{
UNA REVISIÓN DE LOS PROCESOS DE SUFOSIÓN O PIPING EN ESPAÑA
}

\author{
J. M. GARCÍA-RUIZ*
}

Instituto Pirenaico de Ecología, CSIC Campus de Aula Dei, Apartado 13.034, 50080-Zaragoza, España.

\begin{abstract}
RESUMEN. Se revisan los estudios que hacen referencia al desarrollo de procesos de sufosión o piping en España. La mayor parte de los trabajos se centran en ambientes semiáridos de la Depresión del Ebro y del Sureste de España, donde llegan a formarse redes subsuperficiales de drenaje muy complejas, dando lugar ocasionalmente a grandes colapsos que a su vez favorecen la evolución de cárcavas. En muchos casos el piping se asocia a la presencia de badlands. La sufosión tiene especial relevancia en suelos y materiales poco consolidados sobre afloramientos de rocas blandas (margas y lutitas), con abundantes arcillas expansivas y sodio, lo que reduce la coherencia de los materiales. La existencia de fuertes gradientes hidráulicos (asociados al aterrazamiento de campos de cultivo o a la incisión por parte de cárcavas) es un factor imprescindible. Muchos de los problemas relacionados con la sufosión se originan en antiguos campos de cultivo y, a veces, en campos regados. En áreas de montaña se ha citado la presencia de redes de drenaje subsuperficiales relacionadas con madrigueras y raíces muertas, aunque no conducen al desarrollo de cárcavas.
\end{abstract}

\section{A review of piping processes in Spain}

ABSTRACT. The papers referred to the development of piping processes in Spain are reviewed. Most of papers are focused on semi-arid environments of the Ebro Depression and South-East Spain, where large collapses occur favouring the evolution of gullies. In many cases, piping is related to the presence of badlands. Piping is particularly relevant on soils and poorly consolidated materials on soft rocks (marls and lutites), with abundant expansive clays and high sodium content. The existence of strong hydraulic gradients (linked to bench-terracing of cultivated soils, or to gully incision) is a necessary factor. Many of the problems related to piping are generated in old cultivated fields or even in irrigated fields. In mountain areas the presence of subsurface drainage networks has been cited as a consequence of the presence of micro-mammals burrowing or dead roots, though they do not lead to the development of badlands. 
Palabras clave: sufosión, drenaje subsuperficial, badlands, cárcavas, abandono de tierras. Key words: piping, sub-superficial drainage, badlands, gullies, land abandonment.

Correspondencia: Instituto Pirenaico de Ecología, CSIC, Campus de Aula Dei, Apartado 13.034, 50080 Zaragoza, España. E. mail: humberto@ipe.csic.es

\section{Introducción}

Se entiende por sufosión o piping todo un conjunto de procesos hidromorfológicos que favorecen el desarrollo de conductos subsuperficiales en materiales generalmente poco coherentes, tanto suelos como depósitos no consolidados (Jones, 2004), o incluso directamente sobre litologías friables. Estos conductos, que tienden a desarrollarse a menos de 5 metros de la superficie, evolucionan por colapso hacia hoyos o agujeros de distinto diámetro y, ocasionalmente, hacia un paisaje caótico en el que tales hundimientos forman parte de formaciones más o menos extensas de badlands (Brian y Yair, 1982). De hecho, los badlands aparecen frecuentemente acompañados por procesos de sufosión o piping, que a la vez contribuyen a acelerar el abarrancamiento y la evolución del paisaje hacia modelados ruiniformes (Brian y Yair, 1982), hasta el punto que se ha afirmado que los conductos o pipes pueden ser tanto una causa como una consecuencia de la evolución de las cárcavas (Jones, 2004). En las páginas siguientes se emplearán indistintamente los términos sufosión o piping. Hoy se considera que la sufosión contribuye localmente y de manera muy destacada a la degradación del suelo y a la exportación de sedimento (García-Ruiz y López-Bermúdez, 2009), a la vez que tiene localmente una importante función en la respuesta hidrológica frente a eventos pluviométricos o a la fusión de la nieve (Jones, 1994). La erosión en túnel, sufosión o piping fue descrita por primera vez en las regiones semiáridas (véase especialmente la síntesis de Brian y Yair, 1982), y más tarde en regiones húmedas (Jones, 1988).

En las páginas siguientes se resume la historia de los estudios sobre sufosión en España y se presentan los resultados más destacados, poniendo especial énfasis en las relaciones existentes entre sufosión y determinadas prácticas agrícolas.

\section{La historia de los estudios sobre sufosión en España}

En España existe ya un número relativamente elevado de estudios que reflejan la diversidad de condiciones en que se produce este tipo de procesos de erosión. Probablemente el primer trabajo que cita específicamente la ocurrencia de grandes colapsos en rocas blandas es el de Cano García (1975), referido a la Depresión de Guadix, donde llega a hablar de "karst mecánico" o "pseudokarst", debido a la presencia de formas muy 
similares a las de los paisajes kársticos. Este trabajo contiene en esencia toda la información necesaria para entender la formación y evolución de un paisaje dominado por la sufosión, que en un estadio muy avanzado acabaría desarrollando un complejo sistema de badlands y, finalmente, por desmantelamiento de este último, un nuevo nivel de glacis. Van Zuidam (1976) también cita la presencia de frecuentes hundimientos en los alrededores de Zaragoza. En un artículo de Gallart (1979), centrado en la Conca d'Odena, sector oriental de la Depresión del Ebro, se incluye la sufosión entre los procesos que condicionan la rápida evolución del paisaje, destacando la ocurrencia de colapsos por sufosión y de cortos cañones formados por el desplome de galerías subsuperficiales. La vinculación entre sufosión y badlands fue puesta de relieve por Harvey (1982) en el Sureste de España. La Reunión sobre Procesos Actuales en Geomorfología que tuvo lugar en Jaca en 1984, representó un impulso para los estudios sobre Geomorfología dinámica o de procesos, contando con un estudio de Gutiérrez-Elorza y Rodríguez-Vidal (1984) sobre sufosión en la Depresión del Ebro, que es el primero que incluye ese término en el título. Por entonces, Lasanta (1985) fue el primero en registrar su presencia en campos de regadío de La Rioja, donde más tarde se llevaron a cabo diversas pruebas experimentales para comprobar la dinámica hidrológica y el transporte de sedimento en una red de conductos subsuperficiales durante los momentos de riego (García-Ruiz et al., 1986 y 1998).

El estudio de la sufosión en España se halla estrechamente asociado a ambientes semiáridos, donde ocurre de manera muy común en determinados contextos geomorfológicos y edáficos. En la Depresión del Ebro destacan los trabajos de Gracia Prieto (1986) en las Bardenas orientales, y los estudios muy detallados de Gutiérrez et al. (1988 y 1997), con análisis de las características físicas de los materiales y de su contexto geomorfológico. A unos 15 kilómetros al sur de Zaragoza se ha estudiado la relación entre el desarrollo de piping y la evolución de la cabecera de una cárcava (Ries y Marzolff, 2003). En el mismo sector se estudió la evolución de los fenómenos de sufosión en relación con las prácticas agrícolas ( Barrón et al., 1994).

En el Sureste de España destacan los trabajos de López Bermúdez y Torcal Sáinz (1986), López-Bermúdez y Romero-Díaz (1989) y los más recientes de RomeroDíaz et al. (2007 y 2009), en los que se refleja la gran influencia de los cultivos y su posterior abandono en el desarrollo de grandes redes de drenaje subsuperficiales y su correspondiente colapso. El carácter dispersivo de los suelos afectados por sufosión fue estudiado de forma específica por Faulkner et al. (2003) en Vera, Almería, donde conductos subsuperficiales y colapsos habían sido citados y cartografiados previamente por Calvo-Cases et al. (1991).

En sedimentos aluviales existen, en cambio muy pocos trabajos, debido a la menor eficacia de la sufosión. Cabe citar la presencia de conductos de notable tamaño en el escarpe de una terraza del río Gállego cerca de Zaragoza (Gutiérrez Elorza y Rodríguez Vidal, 1984), y el estudio de Ternan et al. (1998) en la raña del interfluvio entre los ríos Jarama y Sorbe, al oeste de la provincia de Guadalajara. 
Finalmente, se ha citado su presencia en ambientes de montaña húmeda (GarcíaRuiz y Puigdefábregas, 1982), aunque no existe ningún estudio específico, seguramente debido a su menor espectacularidad en comparación con los ejemplos observados en el Sureste español o en la Depresión del Ebro. De hecho, no se ha citado la presencia de sufosión asociada a la ocurrencia de badlands en áreas de media o alta montaña. Varios trabajos citan, no obstante, la presencia de conductos subsuperficiales en bosques de la Sierra de la Demanda (Sistema Ibérico), donde se ha señalado que la mayor parte de la circulación hídrica es subsuperficial, en gran medida a través de conductos o pipes causados por madrigueras de micromamíferos o raíces muertas (García-Ruiz y ArnáezVadillo, 1984; Arnáez-Vadillo y García-Ruiz, 1984; Arnáez Vadillo, 1987). A diferencia de los pipes de ambientes semiáridos, muchos de los pipes de montaña mantienen circulación sostenida a lo largo del año.

\section{Los factores determinantes de la sufosión}

La formación de conductos subsuperficiales se debe a la interacción de varios factores generalmente coincidentes en un mismo territorio, de manera que la ocurrencia de uno de ellos es insuficiente para explicar el inicio y desarrollo de una compleja red de drenaje que favorece una rápida evacuación de la escorrentía. Entre tales factores destacan las características del suelo o del regolito, las características locales de la topografía y otros factores que favorecen la canalización del flujo.

(i) La razón principal de la ocurrencia de piping está en las características físicoquímicas del suelo: presencia de arcillas expansivas, elevados valores de SAR (Sodium Adsorption Ratio) y de sodio intercambiable (ESP: Exchangeable Sodium Percentage) (Gutiérrez et al., 1997; Ternan et al., 1998; Desir y Marín, 2009). Altos valores de SAR y ESP son suficientes para definir un lugar como altamente susceptible al piping, como ocurre en la Estación Experimental de las Bardenas (Gutiérrez Elorza et al., 1995). Cerca de la ciudad de Huesca, en el municipio de Lupiñén, el sodio llega a representar entre el 80 y el $90 \%$ del total de cationes, explicando, junto a otros factores, el desarrollo de colapsos de gran envergadura, con diámetros de hasta $35 \mathrm{~m}$ y profundidad de hasta 20 m (Gutiérrez et al., 1988). La sufosión coincide, por lo tanto, con materiales poco coherentes, de baja resistencia a la erosión hídrica (con alto contenido en limos y arcillas, es decir en afloramientos de margas y lutitas) con elevado contenido en sales. Estos materiales pueden verse afectados por la disolución del sodio, de manera que se acelera la pérdida de coherencia y la fácil exportación de materiales finos en cuanto se inician conductos por los que fluye el agua lateralmente. Se trata, por lo tanto, de los mismos factores que, en buena parte, explican la formación de badlands (Gutiérrez et al., 1988). Por otro lado, al haber un predominio de arcillas expansivas, se favorece el agrietamiento y la canalización de la escorrentía hacia el interior del suelo siguiendo unas líneas preferentes de flujo subsuperficial (Cano García, 1975; Gutiérrez et al., 1997). Los minerales del grupo esmectita, como la montmorillonita, se caracterizan por sus elevadas tasas de expansión y contracción, aumentando la susceptibilidad a la 
desecación, agrietamiento e infiltración (Jones, 2004). En badlands de la provincia de Alicante, Cerdà y Bodi (2009) señalan que la infiltración es muy variable espacialmente debido a la presencia de macroporos y grietas que favorecen la infiltración del agua más en unos lugares que en otros, creando condiciones favorables para la sufosión, tal como apuntan también Romero-Díaz et al. (2007). En ocasiones, sin embargo, la presencia de altos valores de SAR y ESP no determinan la ocurrencia de intensos procesos de sufosión, como han señalado Desir y Marín (2007) en las Bardenas Reales, sobre todo en laderas pendientes donde la escorrentía tiende a fluir en superficie.

Las condiciones precedentes se alcanzan tanto en suelos y regolitos desarrollados directamente sobre lutitas y margas, como en materiales acumulados en fondos de valle, donde forman rellenos de notable potencia. Este último caso es frecuente en la Depresión del Ebro y en el Sureste de España, donde procesos históricos y subactuales de erosión han dado lugar a acumulaciones importantes de materiales desplazados desde las laderas (ver, por ejemplo, Burillo et al., 1985; Peña et al., 1993; Arauzo y Gutiérrez, 1995). Gutiérrez Elorza y Rodríguez Vidal (1984), al estudiar la sufosión en extensos derrames holocenos de la Hoya de Huesca encontraron que estos depósitos contienen fragmentos de cerámicas medievales, lo que indica una edad postmedieval para la culminación de tales derrames y aún más reciente para la instalación de drenajes subsuperficiales y de los consiguientes colapsos.

(ii) La presencia de niveles menos permeables en profundidad favorece la circulación horizontal y la evolución de conductos inmediatamente por encima del nivel impermeable. En suelos afectados por sufosión en La Rioja se ha comprobado este descenso de la permeabilidad en profundidad, por transporte vertical de arcillas. Los conductos de sufosión tendían a localizarse en el nivel en que se producía un marcado cambio de textura (García-Ruiz et al., 1986). Este mismo fenómeno también se ha observado en cuencas neógeno-cuaternarias del sureste de España (López-Bermúdez y Torcal-Sáinz, 1986; López Bermúdez y Romero Díaz, 1989), con una dificultad creciente de la infiltración hacia niveles inferiores por una disminución de la porosidad en profundidad.

(iii) La existencia de un gradiente hidráulico es imprescindible para la formación y colapso de conductos subsuperficiales. Es decir, se requiere un importante salto topográfico que aumente la velocidad del flujo y, por lo tanto, que facilite el arranque de partículas en el interior del suelo y su transporte. Por ello, los mejores ejemplos de colapsos aparecen cerca de barrancos que han experimentado un proceso de encajamiento, y también cerca de los bordes externos de bancales artificiales, en los que hay un importante desnivel. En ocasiones, incluso, se ha detectado la presencia de piping en orillas fluviales, sobre depósitos de terraza, como señalaron Gutiérrez Elorza y Rodríguez Vidal (1984) en el río Gállego, cerca de la localidad de Peñaflor. Esto sugiere la existencia de un gradiente hidráulico muy fuerte, capaz de dotar de alta energía a los flujos subsuperficiales, de manera que puedan arrastrar gravas.

(iv) Cano García (1975), Gutiérrez Elorza y Rodríguez Vidal (1984) y López- Bermúdez y Torcal-Sáinz (1986) señalan también la importancia de los fuertes contrastes 
pluviométricos estacionales típicos de los ambientes semiáridos. Los largos periodos sin lluvias favorecen la desecación y el agrietamiento, que más adelante dirigen la infiltración durante precipitaciones muy intensas, contribuyendo a una rápida erosión en profundidad.

(v) Por último, la presencia de madrigueras, raíces de plantas o grietas de tensión (Harvey, 2004) por las que circula el agua constituye un factor favorable adicional, al dirigir la circulación subsuperficial por conductos previamente establecidos que pueden ampliar su diámetro más rápidamente. Borghi et al. (1990) estudiaron en ambiente de pastos subalpinos del Pirineo (Los Lecherines, alto valle del Aragón) el suelo removido por roedores, cuantificándolo en más de 7 toneladas por hectárea, extraídas de la construcción de madrigueras que más tarde funcionan como auténticos pipes por los que fluye más fácilmente el agua de fusión de la nieve en primavera.

\section{Algunos resultados destacados}

Harvey (1982) insiste en la estrecha relación existente entre procesos de piping y evolución de badlands en el Sureste de España. Este autor estudia diferentes áreas de cárcavas entre Altea (Alicante) y el Campo de Tabernas (Almería), distinguiendo varios tipos de piping con diferente influencia sobre la morfología de las cárcavas. Los pipes superficiales, de unos pocos centímetros de diámetro, se relacionan con la presencia de un nivel desagregado con un espesor de unos pocos centímetros por debajo de la costra superficial. No tienen aparentemente ningún papel en la evolución de las cárcavas. En cambio, los pipes profundos (tanto si se deben a permeabilidad diferencial entre los diferentes niveles del regolito o del sustrato, como a grietas de tensión que dirigen el desarrollo de pipes) producen grandes colapsos que pueden llegar a alterar el trazado de cárcavas, y más cuando, a semejanza de las uvalas kársticas, conectan varios colapsos entre sí para dar lugar a grandes hundimientos de bordes ondulados (Fig. 1).

Gutiérrez Elorza et al. (1997) han estudiado en detalle los procesos de piping en distintos puntos de la Depresión del Ebro, incluyendo Bardenas y las proximidades de la ciudad de Huesca. En el primer caso el estudio se centra en la evolución de pipes en laderas de arcillas miocenas, donde los conductos suelen ser de pequeño tamaño. La densidad de pipes se correlaciona bien con la densidad de grietas, el porcentaje de illita, el índice de dispersión y la pendiente. En las proximidades de Huesca (Castillo de Orús) el estudio se centra en rellenos sedimentarios de fondo de valle, con una densidad mucho mayor que en Bardenas y con un tamaño muy superior de los colapsos superficiales. La mineralogía muestra un predominio muy marcado de la illita (que llega hasta el $85 \%$ de la fracción arcilla), y los análisis físicos presentan un elevado índice de dispersión. Una evolución muy similar se da también en depósitos no consolidados de fondo de valle en Azlor, muy cerca del caso anterior (Gutiérrez Elorza y Rodríguez Vidal, 1984), donde es evidente la dispersión de arcillas por disolución del sodio. 


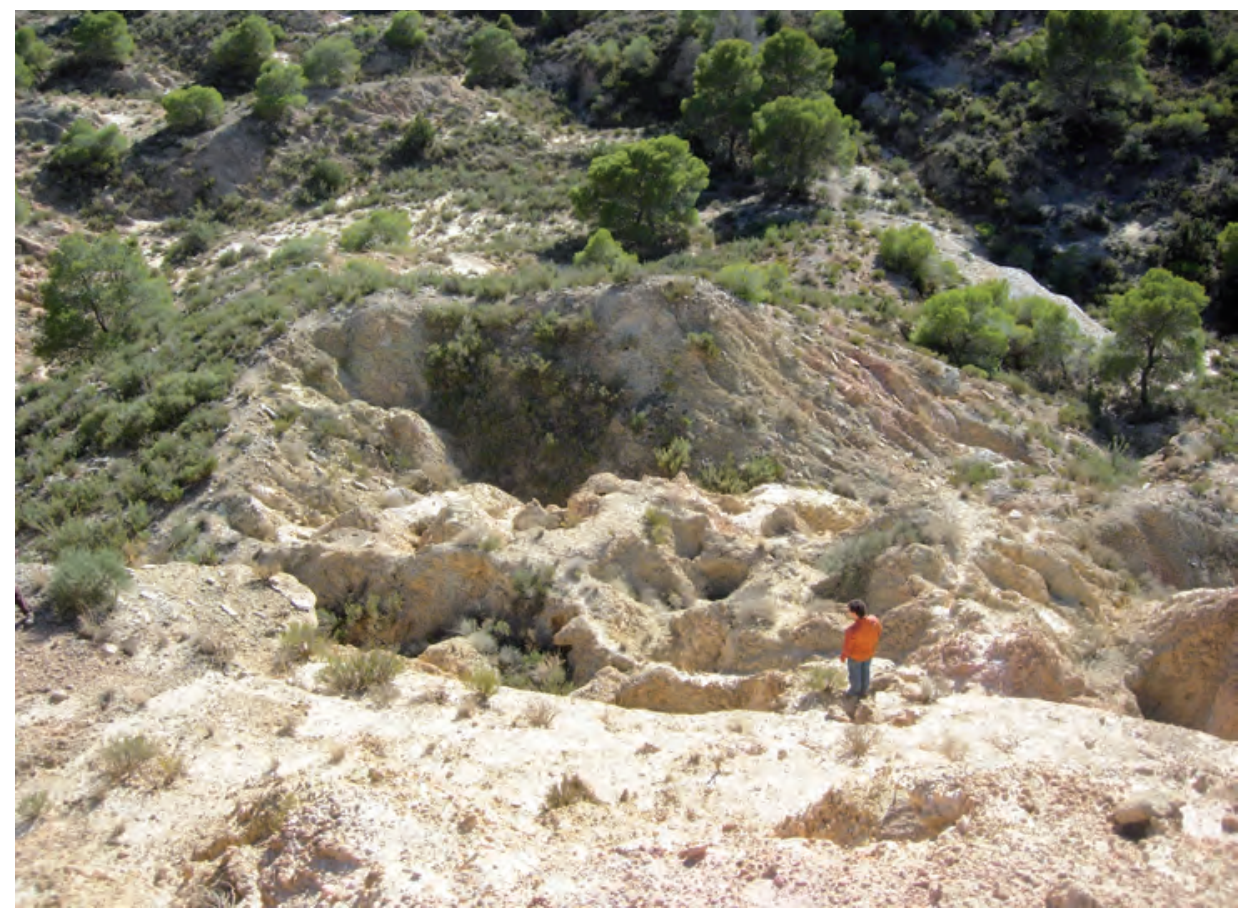

Figura 1. Grandes colapsos en el sector de Jubierre (Monegros, Depresión del Ebro), donde se relacionan con el desarrollo de un paisaje de badlands muy activo.

En Azlor el proceso de formación de pipes es muy activo, con hundimientos de dimensiones hectométricas que son rellenados por los agricultores para frenar su evolución. La mayoría de los colapsos aparece en los bordes de bancales de cultivo, donde el gradiente hidráulico es mayor y, por lo tanto, mayor la energía erosiva de los flujos subsuperficiales. En general, se afirma que el encajamiento de las cárcavas en rellenos de fondo de valle es el factor más importante para facilitar una rápida evolución de los pipes en sus proximidades, al aumentar el gradiente hidráulico (Harvey, 1982; Gutiérrez Elorza et al., 1988). Ternan et al. (1998) tras estudiar la susceptibilidad al piping en la Formación de Raña entre los ríos Jarama y Sorbe (prov. de Guadalajara), señalan que la incisión de cárcavas proporciona el gradiente hidráulico necesario para el desarrollo de una red de pipes. A partir de ahí la evolución es muy rápida por ampliación de pipes, colapsos y caída de paquetes de sedimento en el borde de la cárcava, hasta generar una morfología caótica. Un proceso muy similar ha sido descrito por Gracia Prieto (1986) en las Bardenas orientales, entre Navarra y Zaragoza), afectando a derrames holocenos, con una alta velocidad de formación.

En la misma Depresión del Ebro se ha constatado la estrecha interdependencia entre piping y evolución de cárcavas. Gutiérrez Elorza et al. (1988), al estudiar la distribución de pipes en unos conos aluviales recientes en Lupiñén, cerca de Huesca, 


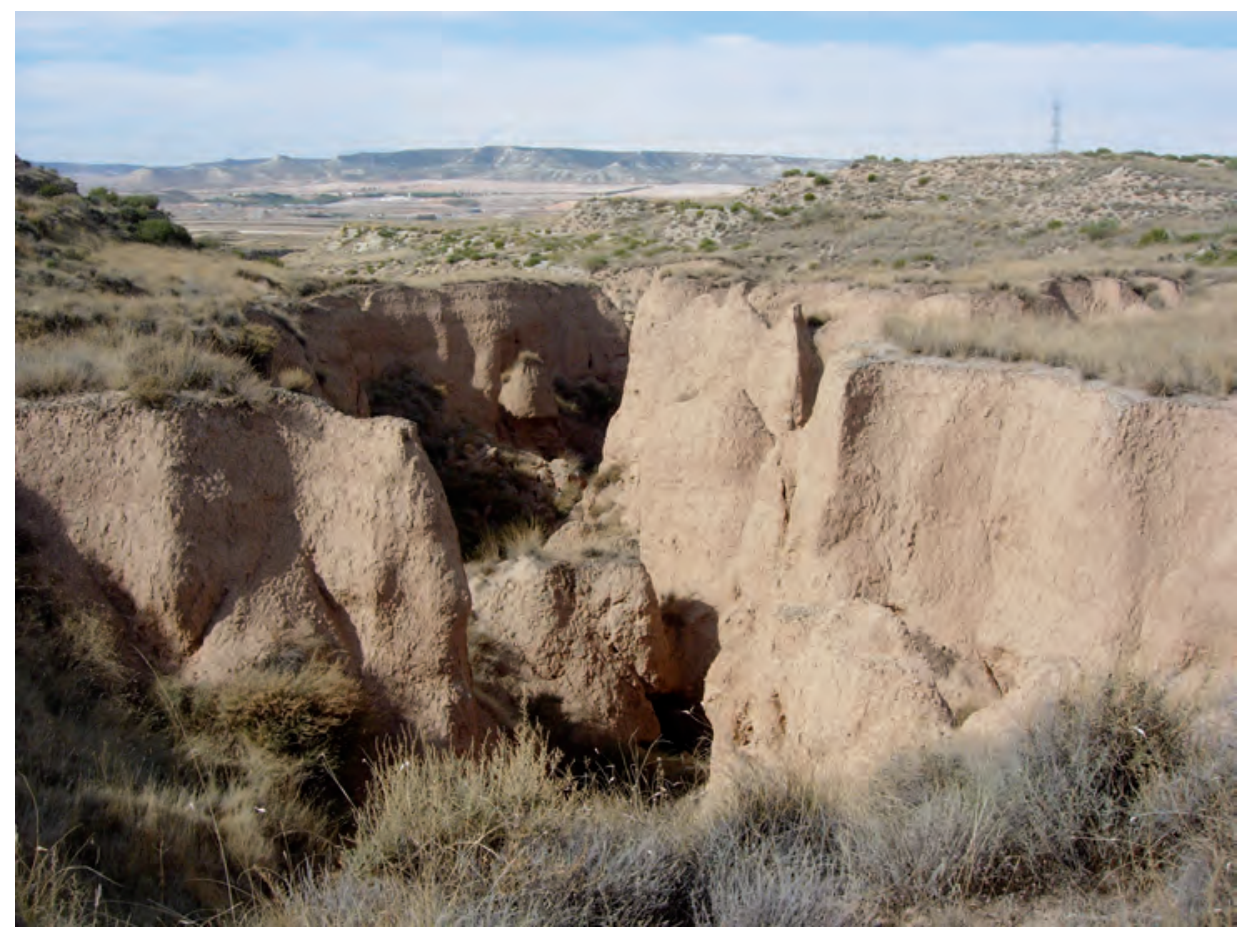

Figura 2. Barranco de Las Lenas, al sur de Zaragoza. La incisión de la cárcava se adapta a los colapsos de la red de drenaje subsuperficial.

concluyen que la densidad de pipes aumenta cerca de las cárcavas y disminuye cerca de los interfluvios. Los pipes controlan la orientación de las cárcavas y estas últimas se desarrollan por colapso de los conductos, hasta el punto de que la forma sinuosa de una cárcava no parece ser resultado de evolución subaérea, sino del colapso de la red de drenaje subsuperficial. En el barranco de las Lenas, Ries \& Marzolff (2003) han comprobado que la cabecera de la cárcava principal está rodeada de colapsos y que la propia forma de esa cárcava muestra festones correspondientes a hundimientos relativamente recientes, de manera que gran parte del retroceso de la cabecera se debe a erosión remontante que captura colapsos y hace más rápido ese retroceso (Fig. 2). A su vez, los procesos de sufosión remontan hacia aguas arriba a medida que se ven incentivados por la progresión de la cabecera de la cárcava. La Fig. 3, referida a un pequeño sector en Vera (Almería), muestra la estrecha vinculación entre pipes y cárcavas. La presencia de pipes dentro de las cárcavas sugiere que estas últimas evolucionan en función de los colapsos de la red subsuperficial; por otro lado, la ocurrencia de pipes fuera de las cárcavas indica que la formación de un notable gradiente hidráulico a medida que se va definiendo la cárcava es un factor importante para explicar la generación de nuevos colapsos que más adelante orientarán la evolución remontante de las cárcavas (Calvo-Cases et al., 1991). En algunos 


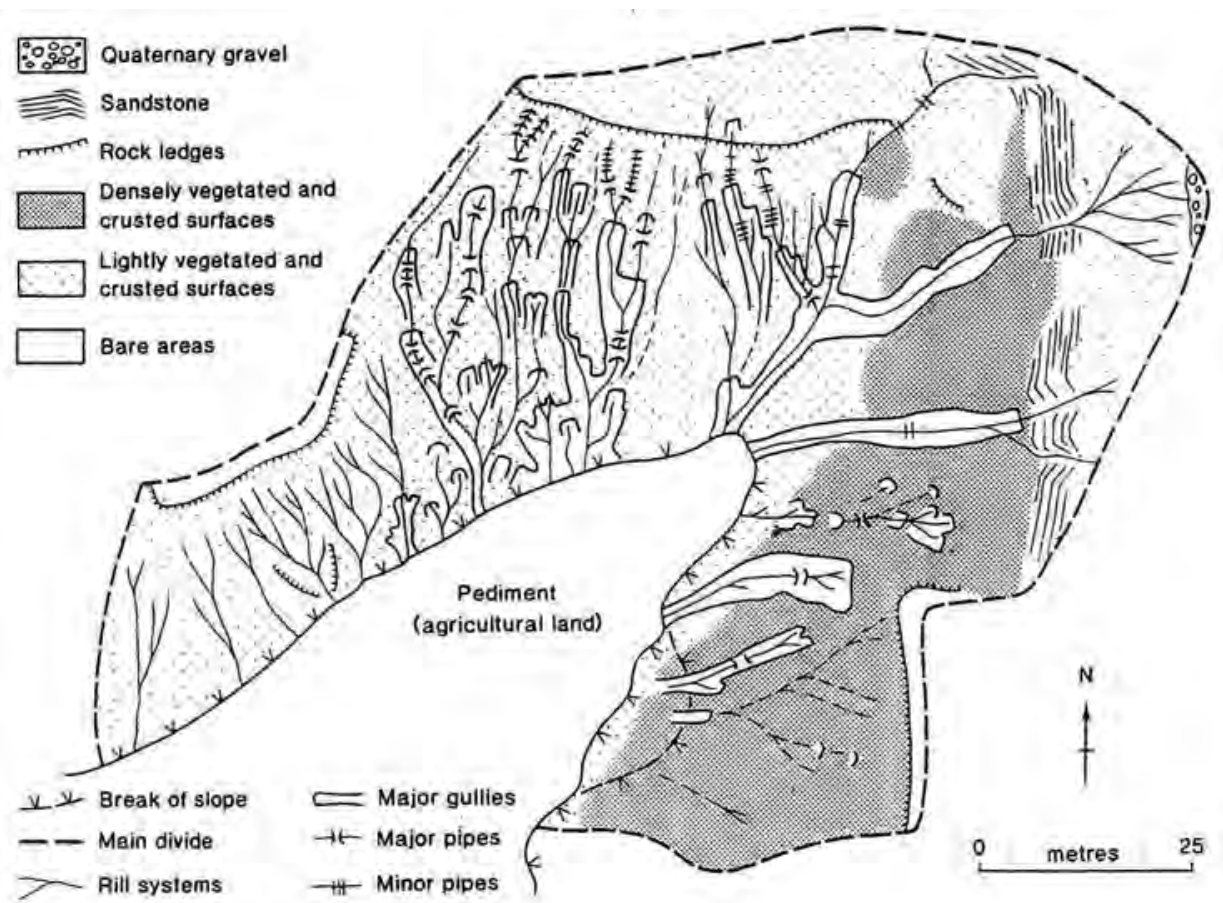

Figura 3. Mapa geomorfológico de un pequeño sector de Vera, Almería, donde se observan las relaciones entre sufosión y evolución de cárcavas. Fuente: Calvo-Cases et al. (1991).

casos se forman auténticos valles ciegos, de manera que se produce una cárcava por hundimiento de una red subsuperficial, pero aguas abajo interrumpen su recorrido bruscamente, al continuar el flujo por vía subsuperficial (Gracia Prieto, 1986). Gallart (1979) se refiere a cañones de sufosión para aludir a galerías que se desploman en gran parte de su trazado, con un canal localmente incidido que aguas arriba termina bruscamente en una pared vertical, sin área de cabecera que lo alimente.

Es importante tener en cuenta que la evolución del piping puede elevar la situación de riesgo de determinados ambientes o de ciertas infraestructuras. Así, el 21 de enero de 2001 una balsa de riego construida en Altorricón (Huesca) en 1999 se rompió en su frente provocando la salida de $300,000 \mathrm{~m}^{3}$ de agua, con un caudal pico de $172 \mathrm{~m}^{3} \mathrm{~s}^{-1} \mathrm{y}$ un transporte de 3500 toneladas de material sólido (Batalla y Balasch, 2001). Gutiérrez et al. (2003) concluyeron que la rotura se debió a sufosión tanto en la cobertera detrítica sobre la que se apoyaba la presa, como en la presa misma, construida con arcillas dispersivas con un alto ESP. La influencia de los pipes en la base de la presa también fue sugerida por Batalla y Balasch (2001). 


\section{Sufosión en áreas de cultivo}

Los procesos de sufosión en campos de cultivo actuales y en campos abandonados pueden ser muy importantes si se dan algunas de las condiciones físicoquímicas y topográficas características de la sufosión. Son frecuentes en los nuevos regadíos de Monegros y Bardenas, donde se benefician de materiales dispersables y de la presencia de sodio en el suelo. En La Rioja han sido estudiados por García Ruiz et al. (1986 y 1997) quienes han comprobado que su evolución está sujeta a factores parecidos a los de los pipes en ambientes semiáridos: elevado gradiente hidráulico (de ahí su presencia casi exclusiva en la proximidades de los saltos entre bancales), sales solubles en el suelo y disminución de la permeabilidad en profundidad. Los suelos son más arenosos en los horizontes superficiales, y más limosos y arcillosos en profundidad, debido a procesos de eluviación por lluvia y riego. Al reducirse la permeabilidad en profundidad, el agua infiltrada tiende a fluir lateralmente siguiendo el gradiente hidráulico y aumentando la erosión subsuperficial. No se han encontrado conductos ni colapsos en campos no abancalados. La mayor densidad y tamaño de los colapsos se presenta en los campos de alfalfa. La razón es sencilla: la alfalfa permanece durante varios años en la misma parcela (un mínimo de 4 ó 5 y un máximo de 9-10 años) sin que el paso anual del arado altere las grietas superficiales por las que el agua penetra en el suelo, o deshaga los canales subsuperficiales. Así, año tras año, el tamaño de los pipes aumenta hasta provocar hundimientos en superficie, que pueden llegar a tener $6 \mathrm{~m}$ de diámetro (Lasanta, 1985), aunque los más comunes oscilan entre 0.5 y 1.5 m de diámetro (Fig. 4).

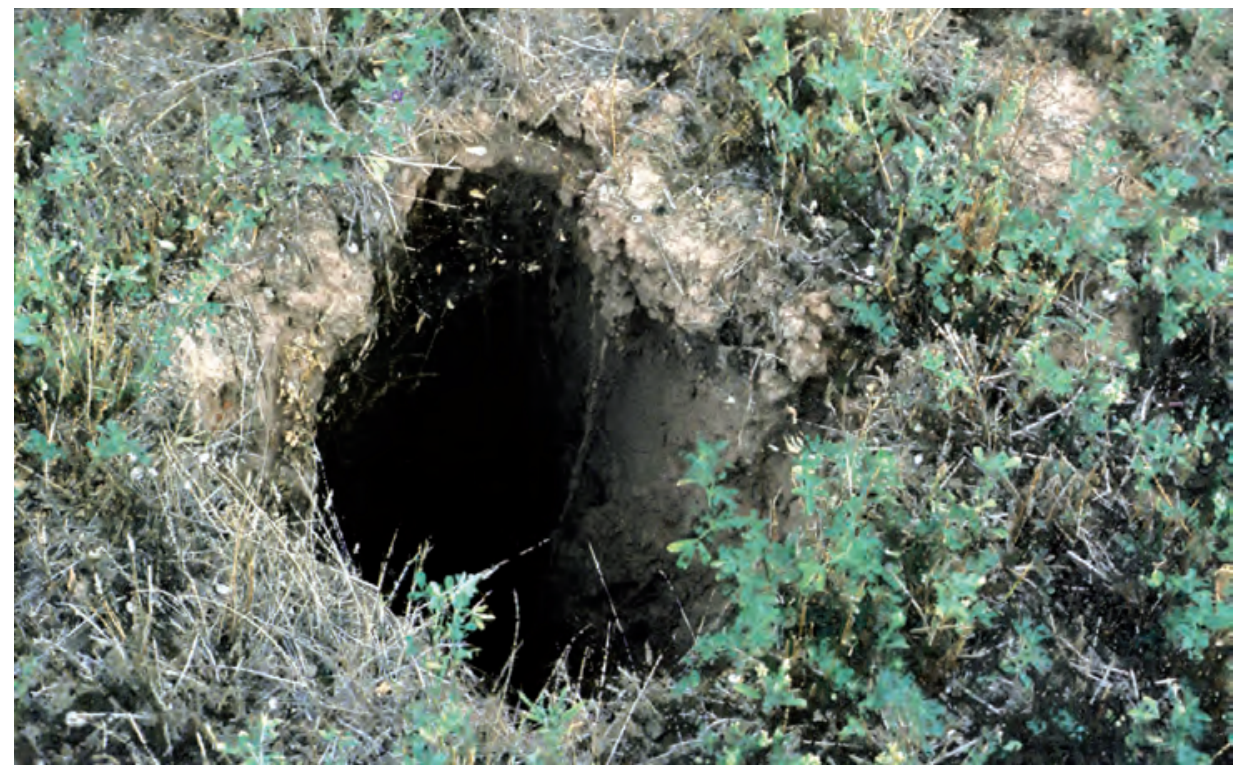

Foto 4. Pequeño conducto vertical (de unos $40 \mathrm{~cm}$ de diámetro) en un campo de regadío en Villamediana (La Rioja) con cultivo de alfalfa. 
Lo contrario ocurre con los campos de cereal, donde cualquier red incipiente de pipes es destruida anualmente por el arado. Por otro lado, los cereales son regados dos veces al año, mientras la alfalfa recibe hasta 10 riegos, lo que acelera mucho el proceso de erosión subsuperficial. García Ruiz et al. (1997) estudiaron el efecto del riego por inundación a lo largo de un año en una parcela de alfalfa en Villamediana de Iregua (La Rioja), regada con aguas con muy baja concentración de sedimento en suspensión y de solutos. La red de pipes evacuó fuera de la parcela en torno al $15 \%$ del agua de riego. La erosión se estimó en torno a $3 \mathrm{Mg} \mathrm{ha}^{-1} \mathrm{año}^{-1}$, lo que sugiere que en campos de regadío la sufosión puede ser el factor más importante de erosión y deterioro del suelo.

Buena parte de estos problemas se debe a la calidad de las aguas de riego y a la consiguiente disolución de sales, como se ha comprobado en algunos perímetros regados de Bardenas, también en la Depresión del Ebro, donde el agua de efluentes de riego multiplicó por 7.4 el contenido de solutos en comparación con el agua de entrada (Lasanta et al., 2001). Alberto y Navas (1987) y Causapé (2010) señalan precisamente que el agua utilizada para riego en la cuenca del Ebro está insaturada en calcita y dolomita, lo que favorece la disolución del carbonato del suelo y, en última instancia, la dispersión y una pérdida de cohesión.

En ambiente de secano, unos 15 kilómetros al sur de Zaragoza, Barrón et al. (1994) sugieren que el abandono de los cultivos ha contribuido a acelerar los procesos de piping, ya que el laboreo en los cultivos de secano tendía a destruir anualmente las redes de pipes que se formaban de manera incipiente. Una vez cesan las labores agrícolas, la evolución de los pipes sigue un proceso ininterrumpido hasta generar grandes colapsos. Por ello, Echeverría et al. (2007) afirman que la evolución de la red de cárcavas y del piping en el Bajo Huerva está relacionado cíclicamente con la política agraria.

En Azlor, al este de Huesca, el proceso de formación de pipes es muy activo, con hundimientos de dimensiones hectométricas que son rellenadas por los agricultores para frenar su evolución. La mayoría de los colapsos aparece en los bordes de bancales de cultivo, donde el gradiente hidráulico es mayor y, por lo tanto, mayor la energía erosiva de los flujos subsuperficiales (Gutiérrez Elorza y Rodríguez Vidal, 1984).

López-Bermúdez y Romero-Díaz (1989) y Romero-Díaz et al. (2007 y 2009) encuentran también relaciones muy estrechas entre el desarrollo de grandes colapsos por sufosión y el abandono de campos de cultivo, en este caso en el sureste de España, siempre en cuencas neógeno-cuaternarias de litología margosa. Estos autores han demostrado que el abandono de campos de cultivo ha propiciado la formación de grandes hundimientos en el plazo de diez años, sobre todo cerca del salto entre bancales (Fig. 5). En muchos casos el abandono del cultivo se produjo en el momento en que se iniciaron los colapsos y parte de los campos dejó de ser aprovechable. Una vez iniciado el proceso y dado el fuerte gradiente hidráulico que se había establecido entre bancales, los conductos subsuperficiales y los colapsos evolucionaron de forma muy rápida (Romero-Díaz et al., 2007) (Fig. 6). La pérdida de suelo por este concepto se ha estimado en más de $550 \mathrm{Mg} \mathrm{ha}^{-1}$ año $^{-1}$, un valor superior al de muchos de los badlands estudiados en España (Nadal-Romero et al., 2008). Esta cifra no debe generalizarse a otros ambientes afec- 


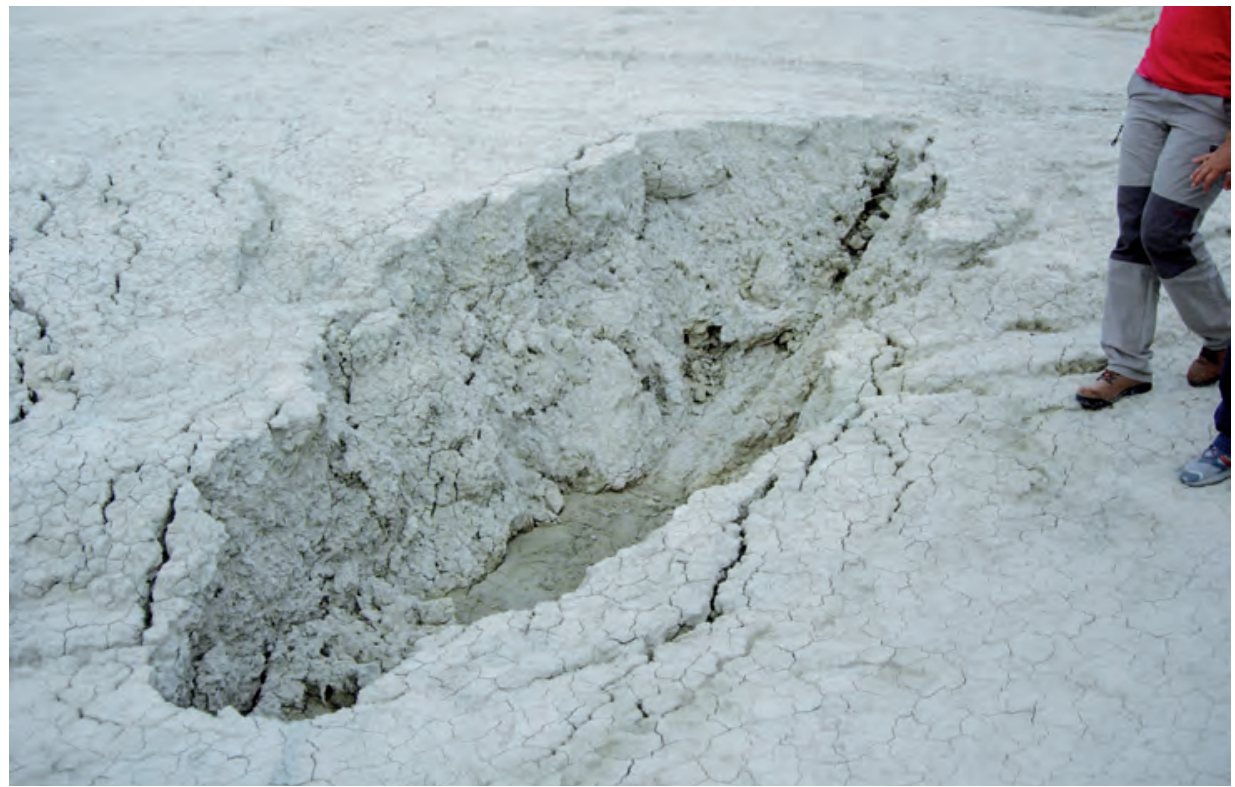

Figura 5. Inicio de colapso en el rellano de un campo abandonado en la cuenca de Mula. Puede deducirse el elevado contenido en margas del suelo.

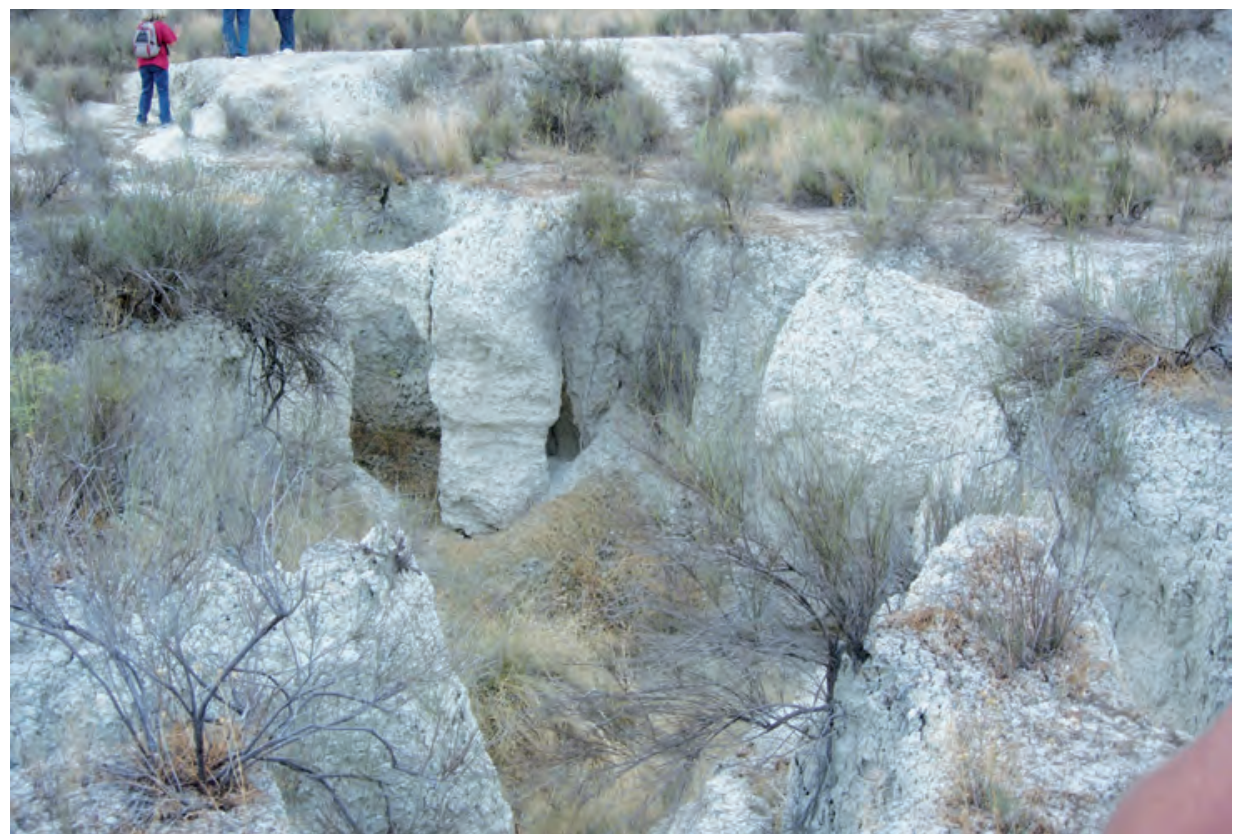

Figura 6. Antiguo campo abandonado en la Cuenca de Mula, donde la elevada densidad de colapsos de la red de drenaje subsuperficial tiende a la construcción de un paisaje de badlands. 


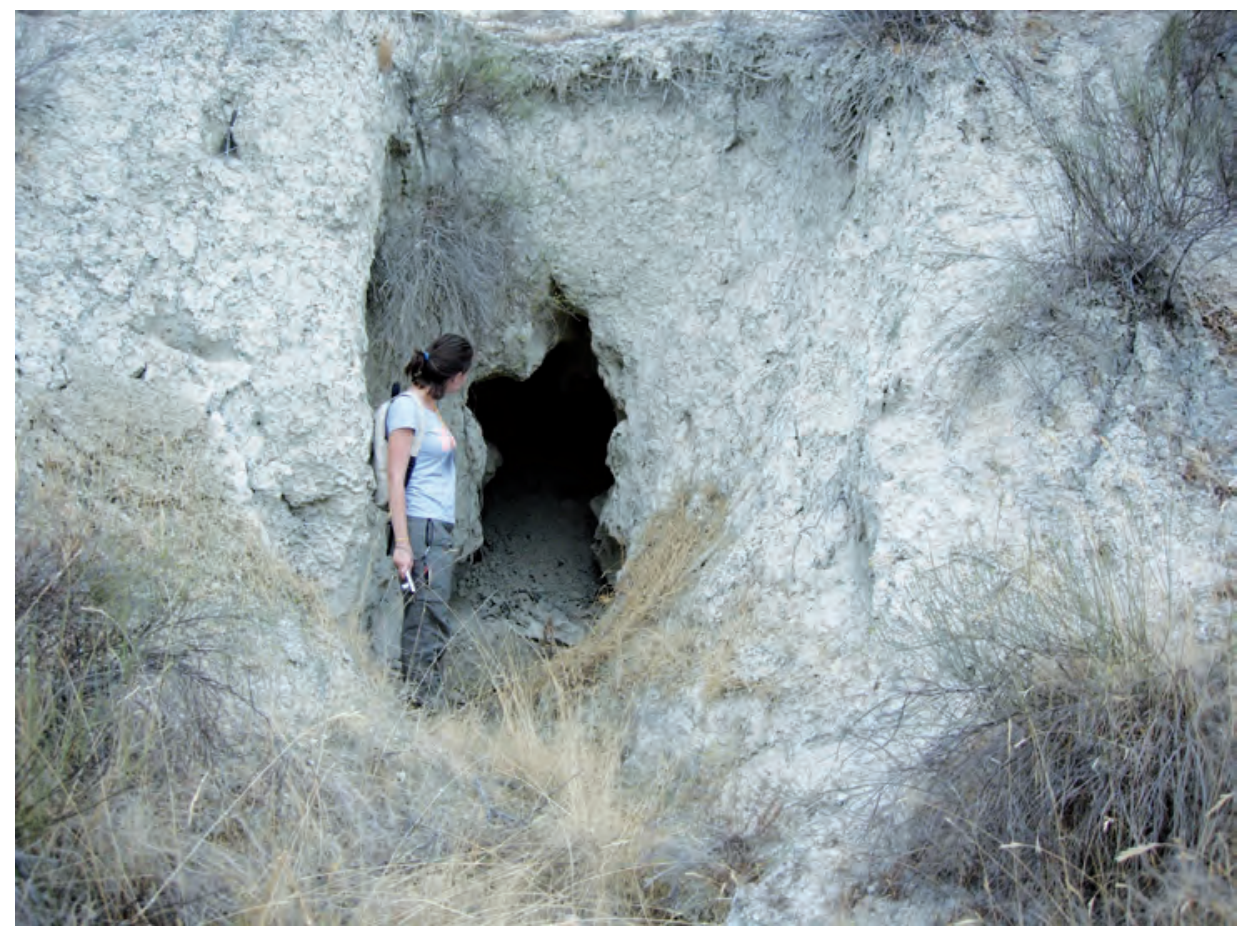

Figura 7. Gran conducto subsuperficial en campo abandonado de la Cuenca de Mula (Murcia), visible por el hundimiento producido en una galería.

tados por sufosión, pues la intensidad de las pérdidas está en relación muy directa con la evolución y magnitud del fenómeno, si bien es cierto que algunos ejemplos de sufosión en la Cuenca de Mula (Murcia) son particularmente espectaculares, tanto por sus dimensiones como por el corto plazo en que se han producido (Fig. 7). Romero-Díaz et al. (2009) apuntan que la profundidad de los hundimientos está muy relacionada con la altura del salto entre bancales (en torno a 2-3 m), aunque se han encontrado colapsos de 7-8 m cuando se han puesto en comunicación directa dos o más bancales. Por esta razón, Romero-Díaz et al. (2007 y 2009) concluyen que, aunque el aterrazamiento contribuye en la mayor parte de los casos a la conservación del suelo en ambientes mediterráneos, en determinadas condiciones puede ser un factor principal de erosión, al aumentar el gradiente hidráulico y favorecer la formación de grandes hundimientos y de cárcavas.

\section{Conclusiones}

Existe un elevado número de estudios sobre sufosión o piping en España, de manera que hoy se conoce no sólo su distribución espacial sino, sobre todo los factores que explican su desarrollo y evolución. La mayor parte de los estudios se han centrado en 
ambientes semiáridos de la Depresión del Ebro y del Sureste de España, donde ocasionalmente se asocian a densas redes de badlands o a la rápida evolución de cárcavas. En esos ambientes la sufosión sólo es posible si coinciden varios factores: (i) suelos y materiales poco consolidados sobre rocas blandas (margas y lutitas), (ii) notable presencia de arcillas expansivas con fuerte tendencia al agrietamiento, (iii) alto contenido en sodio, que favorece la dispersión de las arcillas, y (iv) fuerte gradiente hidráulico en cortas distancias. El desarrollo de redes subsuperficiales de drenaje acaba generando grandes colapsos, de varios metros de diámetro y de profundidad, lo que incentiva la formación de cárcavas que a su vez favorecen la aparición de nuevos conductos y colapsos.

Es interesante constatar que muchos de los mejores ejemplos de piping en España se dan en fondos de valle rellenos de sedimentos en periodos relativamente recientes (Holoceno). La instalación de un barranco o cárcava incidiendo sobre materiales poco consolidados puede ser el punto de partida de un proceso generalizado de sufosión. En algunos casos, más aún en el Sureste de España que en la Depresión del Ebro, la construcción de terrazas de cultivo (con su consiguiente gradiente hidráulico) ha formado grandes colapsos y ha inducido al abandono de los cultivos, favoreciendo una rápida evolución hacia un paisaje ruiniforme y de badlands. También se han citado en campos de regadío, a condición de que estén ligeramente abancalados y se cultiven durante varios años con alfalfa.

Aunque la presencia de pipes es bastante común en ambientes húmedos de montaña, en España no existen estudios específicos sobre este tema, si bien se ha citado su ocurrencia tanto en el Pirineo como en el Sistema Ibérico, donde contribuyen a una rápida conexión entre laderas y cauces en periodo de lluvias o de fusión de nieve.

\section{Agradecimientos}

Este trabajo se enmarca en los resultados obtenidos por los proyectos PROBASE (CGL2006-11619/HID, Consolider), financiado por el Ministerio de Ciencia y Tecnología, ACQWA, financiado por la Comisión Europea, y RESEL, financiado por el Ministerio de Medio Ambiente.

\section{Referencias bibliográficas}

Alberto, F., NAVAS, A., (1987). Caracterización de los niveles de saturación en calcita, dolomita y yeso de las aguas superficiales de la cuenca del Ebro. Anales de Aula Dei,18 (3-4): 199-228.

ARnÁEZ-VAdillo, J., (1987). Formas y procesos en la evolución de vertientes de la Sierra de la Demanda (Sistema Ibérico). Cuadernos de Investigación Geográfica, 13: 1-153. 
ARnÁEZ-VADillo, J., GarcíA-Ruiz, J.M., (1984). Tipos de escorrentía y ritmo de evacuación de sedimentos en la Sierra de la Demanda. Sistema Ibérico. Cuadernos de Investigación Geográfica, 10: 17-28.

Barrón, G., Echeverría, M.T., Ibarra, P., Marco, P., Pérez Cabello, F., (1994). Algunas consecuencias geomorfológicas del uso del suelo agrícola en las últimas décadas. La actividad del piping en el bajo valle del Huerva (Zaragoza, España). En Estudios de Geomorfología en España (Arnáez, J., García Ruiz, J.M., Gómez Villar, A., eds.), Sociedad Española de Geomorfología, pp. 255-266, Logroño.

BATALLA, T.J., BALASCH, J.C., (2001). Interpretación hidrodinámica y sedimentaria de la rotura de la balsa de San Juan (Altorricón, Huesca). Cuaternario y Geomorfología, 15: 109-123.

Borghi, C.E., Giannoni, S.M., Martínez Rica, J.P., (1990). Soil removed by voles of the genus Pitymis in the Spanish Pyrenees. Pirineos, 136: 3-18.

BRIAN, R., YAIR, A., eds., (1982). Badland geomorphology and piping. GeoBooks, 408 pp., Norwich.

Burillo, F., GutiÉrReZ, M., PeÑA, J.L., (1985). Las acumulaciones holocenas y su datación arqueológica en Mediana de Aragón (Zaragoza). Cuadernos de Investigación Geográfica, 11: 193-207.

Calvo-Cases, A., Harvey, A.M., PayÀ-Serrano, J., (1991). Process interactions and badland development in SE Spain. En: Soil erosion studies in Spain (Sala, M., Rubio, J.L., García-Ruiz, J.M., eds.), Geoforma Ediciones, pp. 75-90, Logroño.

CANO García, G.M., (1975). Un ejemplo de karst mecánico en rocas blandas: Las torcas de Guadix. Estudios Geográficos, 138-139: 247-263.

CAusapé VAlenzUela, J.A., (2010). Evaluación y vigilancia agroambiental de los regadios del Ebro: el caso de Bardenas. Publicaciones del Consejo de Protección de la Naturaleza de Aragón, 70 pp., Zaragoza.

Desir, G., MARÍn, C., (2007). Factors controlling the erosion rates in a semi-arid zone (Bardenas Reales, NE Spain). Catena, 71: 31-40.

Desir, G., MARín, C., (2009). Caracterización de la erosión en áreas acarcavadas de la fm. Tudela (Bardenas Reales, Navarra). Cuadernos de Investigación Geográfica, 35 (2): $195-213$.

Faulkner, H., AleXander, R., Wilson, B.R., (2003). Changes to the dispersive characteristics of soils along an evolutionary slope sequence in the Vera badlands, southeast Spain: implications for site stabilization. Catena, 50: 243-254.

GALlaRT, F., (1979). Observaciones sobre la geomorfología dinámica actual en la Conca d'Odena (alrededores de Igualada, Provincia de Barcelona). Actas de la III Reunión Nacional del G.E.T.C., pp. 123-134. 
GARcía-Ruiz, J.M., PuigdefábreGas, J., (1982). Formas de erosión en el flysch eoceno surpirenaico. Cuadernos de Investigación Geográfica, 8: 85-128.

GARcíA-RuiZ, J.M., ARNÁEZ-VAdiLlo, J., (1984). Infiltration, macro-circulation de l'eau et dynamique des versants Dans la Sierra de la Demanda (Système Ibérique, Rioja, Espagne). Documents d'Écologie Montagnarde, 3-4: 445-448.

GARcíA-Ruiz, J.M., LóPEZ-BermúdeZ, F., (2009). La erosión del suelo en España. Sociedad Española de Geomorfología, 441 pp., Zaragoza.

García Ruiz, J.M., LASANTA, T., AlBERTO, F., (1997). Soil erosion by piping in irrigated fields. Geomorphology, 20: 269-278.

García-Ruiz, J.M., LASANTA, T., ORTigOSA, L., ARnÁEZ, J., (1986). Pipes in cultivated soils of La Rioja: origin and evolution. Zeitschift für Geomorphologie Suppl. Bd, 58: $93-100$.

Gracia PRIETO, F.J., (1986). Dinámica erosiva del piping: Un ejemplo en la Depresión del Ebro. Cuadernos de Investigación Geográfica, 12: 11-24.

GutiÉrrez, F., Desir, G., GutiÉRrez, M., (2003). Causes of the catastrophic failure of fan Earth dam built on gypsiferous alluvium and dispersive clays (Altorricón, Huesca Province, NE Spain). Environmental Geology, 43: 842-851.

GuTiÉRrez ElORZA, M., RodríGUEZ VidAL, J., (1984). Fenómenos de sufosión (piping) en la Depresión Media del Ebro. Cuadernos de Investigación Geográfica, 10: 75-83.

Gutiérrez, M., Benito, G., Rodríguez, J., (1988). Piping in badland areas of the Middle Ebro Basin, Spain. Catena Suppl., 13: 49-60.

Gutiérrez, M., Sancho, C., Benito, G., Sirvent, J., Desir, G., (1997). Quantitative study of piping processes in badland areas of the Ebfro Basin, NE Spain. Geomorphology, 20: 237-253.

Gutiérrez Elorza, M., Sancho Marcén, C., Desir Valen, G., Sirvent Mira, J., Benito Fernández, J., CAlvo CASES, A., (1995). Erosión hídrica en terrenos arcillosos y yesíferos de la Depresión del Ebro. Universidad de Zaragoza, 389 pp., Zaragoza.

HARVEY, A., (1982). The role of piping in the development of badlands and gully systems in southeast Spain. En: Badland geomorphology and piping (Bryan, R., \& Yair, A., eds.), GeoBooks, pp. 317-336, Norwich.

Harvey, A., (2004). Badland. En Encyclopedia of Geomorphology (Goudie, A.S., ed.), Routledge, pp. 44-48, London.

JONES, J.A.A., (1988). Modelling pipeflow contributions to stream runoff. Hydrological Processes, 2: 1-17. 
JONES, J.A.A., (1994). Soil piping and its hydrogeomorphic function. Cuaternario y Geomorfología, 8 (3-4): 77-102.

JONES, J.A.A., (2004). Pipe and piping. En: Encyclopedia of Geomorphology (Goudie, A.S., ed.), Routledge, pp. 784-788, London.

LASANTA, T., (1985). Aportación al estudio de la erosión hídrica en campos cultivados de La Rioja. Instituto de Estudios Riojanos, 152 pp., Logroño.

Lasanta, T., Pérez Rontomé, M.C., Machín, J., Navas, A., Mosch, W., Maestro, M., (2001). La exportación de solutos en un polígono de regadío de Bardenas (Zaragoza). Cuaternario y Geomorfología, 15 (3-4): 51-66.

LÓPEZ-BermúdeZ, F., TORCAL-SÁINZ, L., (1986). Procesos de erosión en túnel (piping) en cuencas sedimentarias de Murcia (España). Estudio preliminar mediante difracción de rayos X y microscopio electrónico de barrido. Papeles de Geografía Física, 11: 7-20.

LóPEZ-Bermúdez, F., Romero-DíAz, M.A., (1989). Piping erosion and badland development in South-East Spain. Catena Suppl., 14: 59-73.

NADAL-Romero, E., REGÜÉs, D., LATRON, J., (2008). Relationships among rainfall, runoff and suspended sediment in a small catchment with badlands. Catena, 74: 127-136.

Peña, J.L., Echeverría, M.T., Petit-Maire, N., Lafont, R., (1993). Cronología e interpretación de las acumulaciones holocenas de la Val de Las Lenas (Depresión del Ebro, Zaragoza). Geographicalia, 30: 321-332.

Romero-Díaz, A., Marín-SAnleandro, P., SÁncheZ-Soriano, A., (2009). Procesos de piping en la región de Murcia (Sureste de España). Cuadernos de Investigación Geográfica, 35 (1): 87-117.

Romero-Díaz, A., Marín-SAnleandro, P., SÁncheZ-Soriano, A., Belmonte-SerRATO, F., FAULKNER, H., (2007). The causes of piping in a set of abandoned agricultural terraces in Southeast Spain. Catena, 69: 282-293.

Ternan, J.L., Elmos, A., FitzJohn, C., Williams, A.G., (1998). Piping susceptibility and the role of hydro-geomorphic controls in pipe development in alluvial sediments, Central Spain. Zeitschrift für Geomorphologie, 42 (1): 75-87.

VAN ZUIDAM, R.A., (1975). Geomorphology and archaeology. Evidences of interrelations at historical sites in the Zaragoza region, Spain. Zeitschrift für Geomorphologie, 19: 319-328. 